\title{
LIE ALGEBRAS ALL OF WHOSE MAXIMAL SUBALGEBRAS HAVE CODIMENSION ONE
}

\author{
by DAVID TOWERS
}

(Received 28th March 1980)

Let $\mathfrak{X}$ denote the class of finite-dimensional Lie algebras $L$ (over a fixed, but arbitrary, field $F$ ) all of whose maximal subalgebras have codimension 1 in $L$. In (2) Barnes proved that the solvable algebras in $\mathfrak{X}$ are precisely the supersolvable ones. The purpose of this paper is to extend this result and to give a characterisation of all of the algebras in $\mathfrak{X}$. Throughout we shall place no restrictions on the underlying field of the Lie algebra.

Precisely, the result we shall prove is

Theorem 1. The Lie algebra $L \in \mathfrak{X}$ if and only if $L / \phi(L)=S \oplus R$, where $\phi(L)$ is the Frattini ideal of $L, S$ is a 3-dimensional simple ideal of $L / \phi(L)$ isomorphic to $L_{1}(0)$ (see below), or is $\{0\}$, and $R$ is a supersolvable ideal of $L / \phi(L)$ (possibly $\{0\}$ ).

If $U$ is a subalgebra of $L$ we denote by $U_{L}$ (the core of $U$ ) the largest ideal of $L$ contained in $U$; if $U_{L}=0$ we say that $U$ is core-free. We shall need the following classification of Lie algebras with core-free subalgebras of codimension 1 which is given by Amayo in (1).

Theorem 2. (Amayo (1), Theorem 3.1). Let $L$ have a core-free subalgebra of codimension 1. Then either (i) $\operatorname{dim} L \leqq 2$, or else (ii) $L \cong L_{m}(\Gamma)$ for some $m$ and $\Gamma$ satisfying certain conditions (see (1) for details).

We shall also need the following properties of $L_{m}(\Gamma)$ which are given in (1).

Theorem 3. (Amayo (1)). (i) If $m>1$ and $m$ is odd, then $L_{m}(\Gamma)$ has only one subalgebra of codimension 1 .

(ii) If $m>1$ and $m$ is even, then $L_{m}(\Gamma)$ has precisely two subalgebras of codimension 1.

(iii) $L_{1}(\Gamma)$ has a basis $\left\{u_{-1}, u_{0}, u_{1}\right\}$ with multiplication $u_{-1} u_{0}=u_{-1}+\gamma_{0} u_{1}\left(\gamma_{0} \in F\right.$, $\gamma_{0}=0$ if $\left.\Gamma=\{0\}\right), u_{-1} u_{1}=u_{0}, u_{0} u_{1}=u_{1}$.

(iv) If $F$ has characteristic different from 2 then $L_{1}(\Gamma) \cong L_{1}(0)$.

(v) If $F$ has characteristic 2 then $L_{1}(\Gamma) \cong L_{1}(0)$ if and only if $\gamma_{0}$ is a square in $F$. 
Using the above we can deduce

Lemma 4. Let $L \in \mathfrak{X}$ and suppose that $M$ is a maximal subalgebra of $L$. Then either (i) $\operatorname{dim} L / M_{L} \leqq 2$, or else (ii) $L / M_{L} \cong L_{1}(0)$.

Proof. Clearly $M / M_{L}$ is a core-free subalgebra of $L / M_{L}$ of codimension 1 . Suppose that $\operatorname{dim} L / M_{L}>2$. Then, by Theorem $2, \bar{L}=L / M_{L} \cong L_{m}(\Gamma)$. Furthermore, it follows easily from Theorem 3(i) and (ii) that $m=1$. Suppose that $\bar{L} \not L_{1}(0)$. Theorem 3 (iii), (iv) and (v) implies that $F$ has characteristic 2 and $\bar{L}$ has a basis $\left\{u_{-1}, u_{0}, u_{1}\right\}$ with $u_{-1} u_{0}=u_{-1}+\gamma_{0} u_{1}, u_{-1} u_{1}=u_{0}, u_{0} u_{1}=u_{1}$, where $\gamma_{0}$ is not a square in $F$. But a simple calculation how verifies that the subalgebra spanned by $u_{-1}$ is maximal, contradicting the fact that $L \in \mathfrak{X}$. The result follows.

One more lemma is needed; namely

Lemma 5. Suppose that $L=S_{1} \oplus S_{2}$ where $S_{1}$ and $S_{2}$ are 3-dimensional simple ideals of $L$, each isomorphic to $L_{1}(0)$. Then $L \notin X$.

Proof. Pick a basis $u_{i 0}, u_{i 1}, u_{i(-1)}$ for $S_{i}(i=1,2)$ such that $u_{i(-1)} u_{i 0}=u_{i(-1)}, u_{i 0} u_{i 1}=$ $u_{i 1}, u_{i(-1)} u_{i 1}=u_{i 0}$. It is easily checked that the subalgebra of $L$ spanned by $u_{10}+$ $u_{20}, u_{11}+u_{21}, u_{1(-1)}+u_{2(-1)}$ is maximal.

Proof of theorem 1. (a) Suppose first that $L \in \mathfrak{X}$. If $L$ is solvable, it is supersolvable ((2), Theorem 7), so suppose further that $L$ is not solvable. Factor out $\phi(L)$, so we may assume that $\phi(L)=0$. There is a maximal subalgebra $M$ of $L$ such that $L / M_{L}$ is not solvable (since otherwise $\left(L^{2}\right)^{2}=L^{(3)} \subset \phi(L)=0$ and $L$ is solvable). By Lemma 4 , $L / M_{L} \cong L_{1}(0)$.

Let $K$ be any maximal subalgebra of $L$ and suppose that $M_{L} \not K$. Then $L=M_{L}+K$. Put $B=M_{L}+K_{L}$. Since $L / M_{L}$ is simple, $B=M_{L}$ or $B=L$. The former implies that $M_{L}=K_{L} \subset K$, a contradiction; so $L=B=M_{L}+K_{L}$. Now $L /\left(M_{L} \cap K_{L}\right) \cong$ $\left(M_{L} /\left(M_{L} \cap K_{L}\right)\right) \oplus\left(K_{L} /\left(M_{L} \cap K_{L}\right)\right) \cong\left(L / K_{L}\right) \oplus\left(L / M_{L}\right)$, so $L / K_{L} \neq L_{1}(0)$ (by Lemma 5). Hence $\operatorname{dim} L / K_{L} \leqq 2$, and so $L^{(3)} \subset K_{L} \subset K$. We have proved that either $M_{L} \subset K$ or else $L^{(3)} \subset K$. Thus, $M_{L} \cap L^{(3)} \subset K$ for all maximal subalgebras $K$ of $L$. It follows that $M_{L} \cap L^{(3)} \subset \phi(L)=0$; in particular, $M_{L}^{(3)}=0$ and $M_{L}$ is solvable.

If $M_{L}=0$ we are done. If $M_{L} \neq 0, M_{L} \notin \phi(L)$, and so there is a maximal subalgebra $N$ of $L$ such that $L=M_{L}+N$. As above, $L=M_{L}+N_{L}$. Put $D=N_{L}^{(3)}+M_{L}$. Then $N_{L}^{(3)} \cong$ $D / M_{L}$ which is an ideal of $L / M_{L}$, and so $N_{L}^{(3)}=0$ or else $N_{L}^{(3)} \cong L_{1}(0)$. The former is impossible since this would imply that $L$ were solvable. Hence $D=L$ and $L=S+R$, where $R=M_{L}$ is solvable, $S=N_{L}^{(3)} \cong L_{1}(0)$ and $L^{(3)} \cap R=0$. Furthermore, $S R=S^{2} R \subset$ $S(S R)=S^{2}(S R) \subset L^{(3)} \cap R=0$, giving $L=S \oplus R$. Finally, $R$ is supersolvable by Theorem 7 of (2).

(b) Now suppose that $\bar{L}=L / \phi(L)=S \oplus R$. By Theorem 7.3 of $(3), \bar{L}=(A+B) \oplus S$ where $A=A_{1} \oplus \ldots \oplus A_{n}$ is the sum of the minimal abelian ideals of $\bar{L}$ and $B$ is abelian. Since $R=A+B$ is supersolvable, $\operatorname{dim} A_{i}=1$ for $1 \leqq i \leqq n$. 
Let $M$ be a maximal subalgebra of $\bar{L}$. If $A \not \subset M$, then there is an $A_{i}(1 \leqq i \leqq n)$ such that $A_{i} \notin M$. But then $\bar{L}=A_{i}+M$ and $M$ has codimension 1 in $\bar{L}$. So assume that $A \subset M$.

Suppose that $B \not \subset M$. Then there is an element $b \in B$ such that $b \notin M$. But $B \bar{L} \subset A \subset$ $M$, so $L=M+U$ where $U$ is spanned by $b$. Thus, again, $M$ has codimension 1 in $\bar{L}$.

Finally, if $R \subset M$, it is clear that $M$ has codimension 1 in $\bar{L}$.

Remark. It is clear from the proof of Theorem 1 that for any $L \in \mathfrak{X}$ we can pick a basis $\left\{a_{1}, \ldots, a_{n}, b_{1}, \ldots, b_{m}, u_{-1}, u_{0}, u_{1}\right\}$ such that

$$
u_{-1} u_{0}=u_{-1}, \quad u_{0} u_{1}=u_{1}, \quad u_{-1} u_{1}=u_{0}
$$

and

$$
a_{i} b_{i}=\lambda_{i j} a_{i} \quad \text { for some } \quad \lambda_{i j} \in F \quad(1 \leqq i \leqq n, 1 \leqq j \leqq m),
$$

all other products being zero.

Acknowledgement. The author would like to thank the University of California at Berkeley for their hospitality while this work was being done.

\section{REFERENCES}

(1) R. K. Amayo, Quasi-ideals of Lie algebras II, Proc. London Math. Soc. (3) 33 (1976), $37-64$.

(2) D. W. Barnes, On the cohomology of soluble Lie algebras, Math. Z. 101 (1967), 343-349.

(3) D. A. Towers, A Frattini theory for algebras, Proc. London Math. Soc. (3) 27 (1973), $440-462$.

Department of Mathematics

UNIVERSTTY OF LANCASTER

LANCASTER LA1 4YL

ENGLAND

and

Department of Mathematics

UNIVERSTTY OF CALIFORNIA

BERKELEY

CA 94720

USA 